qubits are used to encode some information, the information is preserved only if none of the photons are lost - something that becomes exponentially more unlikely as the number of qubits required increases. In this respect, Kues and colleagues' use of multilevel encoding, rather than the two-level encoding of qubits, is a smart choice because the same information can be transferred using fewer carriers, which reduces the impact of photon losses.

Another limitation of many quantum photonic systems, including that of Kues et al., concerns the use of a spontaneous nonlinear process to produce the photons used as carriers of the quantum information. This intrinsically probabilistic process causes practical difficulties when synchronizing the operations that comprise a quantum protocol. In addition, the limited rate at which photons are generated, and the low probability that different devices will emit single photons at the same time, make the scaling of these systems rather unfavourable. A promising alternative involves the use of structures called quantum dots ${ }^{9}$, whose atom-like behaviour enables single photons to be generated on demand and at a relatively high rate. However, the efficiency at which these photons are collected still needs to be improved, and the creation of entanglement between photons is much more complicated than in the nonlinear production processes.

The future success of quantum technologies, in terms of outperforming their classical counterparts, will be crucially dependent on the amount of information that can be processed by a quantum platform. Kues and colleagues estimate that their multilevel encoding of two photons could achieve the equivalent of 13 qubits - still far from the hundreds or more that are required to demonstrate the potential of quantum technologies. This limitation is not specific to quantum photonics, but is common to all such current platforms. The next challenge will be to demonstrate a technical breakthrough in one of these platforms or to combine them in a synergistic way to harness the advantages that each can provide.

Roberto Osellame is at the Institute for Photonics and Nanotechnologies (IFN) of the Italian National Research Council (CNR), 20133 Milan, Italy.

e-mail: roberto.osellame@ifn.cnr.it

1. Kues, M. et al. Nature 546, 622-626 (2017).

2. Ladd, T. D. et al. Nature 464, 45-53 (2010).

3. O'Brien, J. L., Furusawa, A. \& Vučković, J. Nature Photon. 3, 687-695 (2009).

4. Tanzilli, S. et al. Laser Photon. Rev. 6, 115-143 (2012).

5. Giovannetti, V., Lloyd, S. \& Maccone, L. Science $\mathbf{3 0 6}$ 1330-1336 (2004)

6. Ursin, R. et al. Nature Phys. 3, 481-486 (2007).

7. Schaeff, C., Polster, R., Huber, M., Ramelow, S. \& Zeilinger, A. Optica 2, 523-529 (2015).

8. Thew, R., Acin, A., Zbinden, H. \& Gisin, N. Quantum Inf. Comput. 4, 93-101 (2004).

9. Somaschi, N. et al. Nature Photon. 10,340-345 (2016).
INFECTIOUS DISEASES

\section{Predictions of virus spillover across species}

\begin{abstract}
Most human infectious diseases are initially transmitted from animals. An analysis of all known mammalian viruses improves our understanding of such cross-species spillover, with potential benefits for public health. SEE LETTER P.646
\end{abstract}

\section{JAMES O. LLOYD-SMITH}

I $\mathrm{n}$ ancient Rome, priests divined the future by examining animal entrails. Today, scientists attempt to predict the emergence of human pandemics by surveying the pathogens carried by animals. Most infectious human diseases, including newly emerging ones, involve pathogens initially transmitted from other animals (these diseases are called zoonoses). Viral zoonoses, such as HIV, pandemic influenza and Ebola, are particularly concerning, given their track record of devastation. On page 646 , Olival et al. ${ }^{1}$ provide the most comprehensive view yet of past, present and future virus-sharing between humans and other mammals.

Efforts to understand the drivers of zoonotic risk have been dogged by concerns about biases arising from uneven research focus across species and regions, and by challenges in untangling alternative hypotheses. For instance, are we at greater risk of zoonoses from apes, which share our genes, or from rats, which share our cities? Influential studies $^{2-4}$ have addressed subsets of these issues or particular host types. Olival et al. confront the full challenge head-on, amassing a database of more than 2,800 animal-virus associations that span all known viruses of mammals and all major orders of terrestrial mammals. The authors used statistical models to attempt to assess all hypotheses at once, while controlling for uneven research efforts. Their work paints a far-reaching picture of the factors that govern how many viruses (total and zoonotic) are carried by mammalian hosts.

Pleasingly, this broad canvas consolidates many earlier findings, often adding nuance. For instance, a host's relatedness to humans was known to affect its propensity to carry zoonotic viruses ${ }^{2}$, but the authors' analysis shows that the detectable effect arises almost entirely from varying patterns of virus carriage between primates. Bats, primates and rodents carry the highest proportions of zoonotic viruses, but only bats carry significantly more than other species after controlling for confounders, reinforcing an earlier finding ${ }^{3}$. An analysis of the viral traits linked to zoonotic potential (aimed at predicting whether a newly discovered virus can infect humans) confirmed that an ability to replicate in the cell cytoplasm increases such potential ${ }^{5}$. This analysis also solidified the intuitive idea that viral generalists, those capable of infecting a wide range of animals, are most likely to be able to infect humans.

The study's most ambitious aim was to predict where and in which host species we might find 'missing' zoonoses - those that exist, but have not yet been detected. The authors extrapolated their models to predict the number of viruses that would be found per host species if all hosts were subject to elevated research efforts, then subtracted from this the number already known for each host (echoing a strategy used to predict future zoonotic diseases from rodents ${ }^{4}$ ). This approach yielded enticing predictions of the species and regions that are most likely to harbour missing zoonoses, such as bats in northern South America and carnivores in East Africa. Although caveats apply, and the authors detected biases in their predictions for some regions, these findings could guide future investment in global virological surveys.

Olival and colleagues must be commended for their robust, methodical approach to tackling this immense problem, the unruly complexity of which is arguably matched only by its value for public health. The authors have brought order to our understanding of virus sharing between humans and other mammals. But the potential of these findings to shape policy and research priorities demands a clear-eyed view of the challenges ahead.

The study aimed to predict zoonotic spillover, but each word in this phrase bears careful examination. First, predict. The researchers considered total virus numbers per species, the proportion of viruses that are zoonotic per species and the zoonotic potential of each virus. For each of these outcomes, they produced models to explain observed patterns (taking into account various biological factors, as well as research effort), which can be pared down to models that predict outcomes for unstudied species (considering only biological factors, because research effort has no effect on true outcome). The authors are exemplary in stating the explanatory power of each model, including the lower power of predictive 


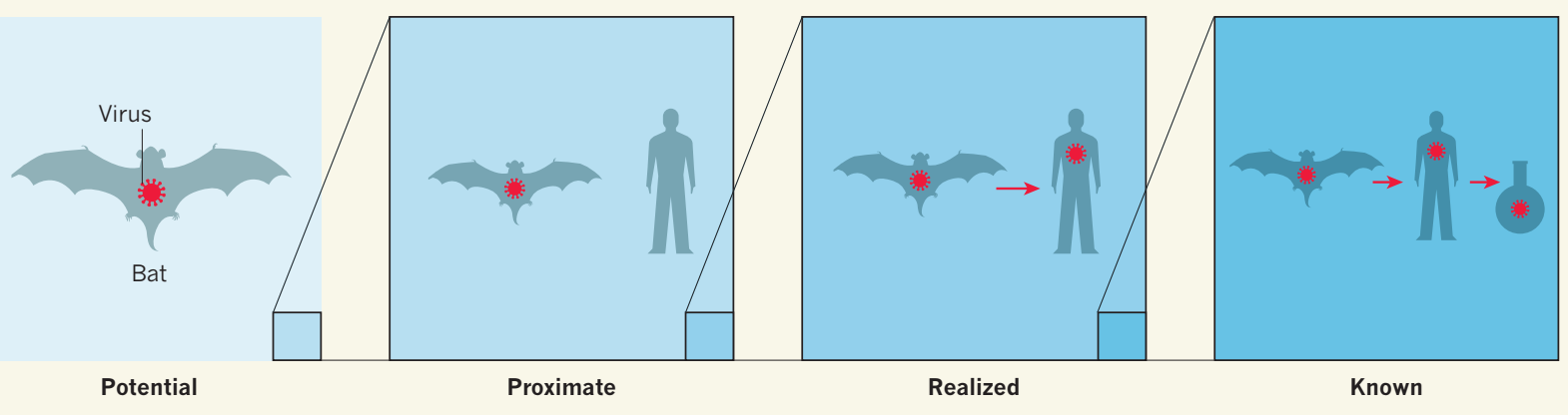

Figure 1 | Grouping viruses that have zoonotic potential. Zoonotic viruses are those that can be transmitted from animals to humans. They can be divided into nested groups on the basis of their success in infecting humans, and our success in detecting them. Potential zoonotic viruses are all animal-borne viruses that are biologically able to infect humans. A subset of these, proximate zoonotic viruses, have the ecological opportunity to infect humans (for instance, because they are carried by animals in close proximity to humans). A further subset, realized zoonotic viruses, actually have infected humans. A final subset, known zoonotic viruses, have been detected in humans and reported in the scientific literature. Olival et al. ${ }^{1}$ have conducted a comprehensive analysis of virus sharing between humans and other mammals, detecting patterns in known zoonotic viruses and predicting patterns in the wider groups.

models. But none of the key outcomes can be predicted with better than 30\% accuracy. We are therefore far from a predictive era in zoonotic disease epidemiology. As the authors state, their predictions are best used to prioritize research and viral-surveillance efforts, not to drive specific policy decisions.

Second, zoonotic. Imagine dividing viruses into nested groups on the basis of their potential - realized or not, and observed or not - to infect humans (Fig. 1). When we say zoonotic, which group do we mean? The authors' analysis of zoonotic potential addresses viruses that may never have encountered humans. Their missing zoonoses include viruses that have infected humans, or may imminently do so, but are not yet recognized. Meanwhile, existing data on zoonotic viruses cover only those known to have infected humans. How well can we project patterns from known zoonoses onto broader groups, given that host and viral traits can differ systematically between them? For instance, viruses that cause more-severe disease are more likely than others to be known, all else being equal. Olival and colleagues' predictions are, necessarily, based on current knowledge. They provide a benchmark against which future data can be compared to chart possible biases arising from projecting trends across these (perhaps dissimilar) groups.

Finally, spillover. The paper addresses whether a given virus is zoonotic and so can spill over from animals to humans, but this is quite different from the quantitative risk of spillover. For example, Olival and colleagues' analysis would not distinguish between Lassa virus (which spills over tens of thousands of times annually ${ }^{6}$ ) and Lujo virus (which has only ever spilt over once, to our knowledge ${ }^{6}$ ). Systematic study of quantitative spillover risks will require approaches that integrate the relevant mechanisms, which occur at scales of molecules to landscapes ${ }^{7}$.

Looking forward, Olival et al. call for investment in viral surveillance, which would expand our knowledge of potential zoonoses particularly if it involves epidemiological metadata and rigorous data-sharing. But although most pandemics are zoonoses, most zoonoses do not cause pandemics, so it is essential not to invest in broad, shallow surveys at the expense of understanding what determines pandemic potential. The crucial factor for a pandemic is human-tohuman transmission, which is governed by viral traits $^{8}$ and population susceptibility ${ }^{9}$ and mobility ${ }^{10}$. Gaining insights into transmissibility will require in-depth field and laboratory studies, combined with the development of quantitative methods to integrate the diverse data streams involved. In this endeavour, datadriven mechanistic models might end up being the new animal entrails.

James O. Lloyd-Smith is in the Department of Ecology \& Evolutionary Biology, University of California, Los Angeles, Los Angeles,
California 90095-7239, USA, and at the Fogarty International Centre, National Institutes of Health, Bethesda, Maryland. e-mail:jlloydsmith@ucla.edu

1. Olival, K. J. et al. Nature 546, 646-650 (2017).

2. Davies, T. J. \& Pedersen, A. B. Proc. R. Soc. B 275, 1695-1701 (2008).

3. Luis, A. D. et al. Proc. R. Soc. B 280, 20122753 (2013).

4. Han, B. A., Schmidt, J. P., Bowden, S. E. \& Drake, J. M. Proc. Natl Acad. Sci. USA 112, 7039-7044 (2015).

5. Pulliam, J. R. \& Dushoff, J. J. Infect. Dis. 199, 565-568 (2009).

6. Bausch, D. G. et al. in Viral Infections of Humans: Epidemiology and Control (eds Kaslow, R. A. et al.) 147-171 (Springer, 2014)

7. Plowright, R. K. et al. Nature Rev. Microbiol. http:// dx.doi.org/10.1038/nrmicro.2017.45 (2017).

8. Geoghegan, J. L., Senior, A. M., Di Giallonardo, F. \& Holmes, E. C. Proc. Natl Acad. Sci. USA 113, 4170-4175 (2016).

9. Gostic, K. M., Ambrose, M., Worobey, M. \& Lloyd-Smith, J. O. Science 354, 722-726 (2016).

10.Pybus, O. G., Tatem, A. J. \& Lemey, P. Proc. R. Soc. B 282, 20142878 (2015).

This article was published online on 21 June 2017.

\section{MATERIALS SCIENCE}

\section{A light-fuelled wave machine}

\section{A polymer that incorporates liquid-crystal components generates continuous mechanical waves when lit by ultraviolet light, with potential applications for energy harvesting and self-cleaning surfaces. SEE LETTER P.632}

\section{YANLEI YU}

$\mathrm{P}$ olymers formed from liquid-crystal molecules are known as liquidcrystal networks (LCNs). They exhibit remarkable properties that allow them to undergo controllable and reversible shape changes in response to external stimuli. When combined with light-sensitive molecules, LCNs can directly convert light energy into 3D motions such as bending, twisting, rotating and oscillating, making them a promising type of material for building actuators (devices that move or control a mechanism or system $)^{1}$. On page 632 , Gelebart et al. ${ }^{2}$ report the first strategy for using an LCN to generate 